\title{
The use of treosulfan and gemcitabine in the treatment of platinum-resistant ovarian cancer
}

\author{
S. HILMAN ${ }^{1}$, P.K. $\mathrm{KOH}^{1}$, S. COLLINS ${ }^{2}$ and R. ALLERTON ${ }^{1}$ \\ ${ }^{1}$ Deansley Oncology Centre, New Cross Hospital, Wolverhampton WV10 OQP; \\ ${ }^{2}$ Cancer Research UK Clinical Trials Unit, School of Cancer Sciences, \\ University of Birmingham, Birmingham B15 2TT, UK
}

Received June 26, 2009; Accepted September 28, 2009

DOI: $10.3892 / \mathrm{ol} 00000038$

\begin{abstract}
The combination of treosulfan and gemcitabine (TG) has been shown to have activity in ovarian cancer. These two agents are thought to be synergistic, with gemcitabine causing the persistence of treosulfan-induced DNA crosslinks. This study aimed to investigate the response rates, survival and toxicity in patients with platinum-resistant ovarian cancer treated with TG. A retrospective case note review of the patients treated with TG was performed in one cancer centre between May 1st, 2000 and November 1st, 2005. Estimates of cumulative survival were obtained using the Kaplan-Meier method. Fortynine patients were identified; median age at diagnosis was 55 years (range, 31-72) and the median follow-up was 45.1 months (range, 12.2-118.3). TG was used as second-, third-, fourth- and fifth-line chemotherapy in 15,19,13 and 2 patients, respectively. Fifteen patients $(30.6 \%)$ had stable disease; $25(51 \%)$, a partial response; 1 (2\%), a complete response and 8 (16.3\%) had progressive disease. Median survival following diagnosis was 45.1 months and the median relapse-free survival was 12 months. The median survival time from the start of TG was 13.7 months with a relapse-free survival of 6.3 months. The median number of cycles given was 7 . The most common toxicity recorded was myelosuppression. There were no treatment-related deaths. TG chemotherapy produced favourable response rates in a heavily pre-treated group of patients with platinum-resistant epithelial ovarian cancer. This doublet warrants further investigation in a phase III trial setting.
\end{abstract}

\section{Introduction}

Ovarian cancer accounts for $5 \%$ of all female types of cancer in the UK, with a lifetime risk of developing the disease in 1 out of 48 for women in England and Wales. In 2005, 6,806 new cases were recorded in the UK (crude incidence rate, 22.1

Correspondence to: Dr Serena Hilman, Weston General Hospital, Grange Road, Uphill, Weston-Super-mare, Somerset, UK

E-mail: serena.hilman@waht.swest.nhs.uk

Key words: ovarian cancer, chemotherapy, gemcitabine, treosulfan, platinum resistance per 100,000), with 4,407 women succumbing to the disease in 2006 (1). Only $15 \%$ of the cases occur in women under 50 years of age. Ninety percent of ovarian types of cancer are epithelial in origin, with germ cell and sex cord-stromal tumours accounting for the remaining $10 \%$ (2).

Patients with platinum-resistant epithelial ovarian cancer have often had multiple lines of chemotherapy. Thus, the aim of further treatment is to improve survival and maintain quality of life. When ovarian cancer progresses within six months of platinum chemotherapy it is considered to be platinumresistant, with poor response rates to further platinum-based therapy. Combination chemotherapy with drugs acting by different mechanisms and exhibiting non-cross resistance may increase the chance of response, as well as prolong progressionfree survival.

Treosulfan is an alkylating agent which is cytotoxic as a result of the formation of DNA interstrand crosslinks and was first reported to have activity in ovarian cancer in the 1970s (3). Gemcitabine is a nucleoside analogue which, through its incorporation into DNA, causes masked chain termination. It also inhibits ribonucleotide reductase, which is required for DNA synthesis and repair.

In combination, treosulfan and gemcitabine have been shown to have activity in uveal melanoma. A phase I trial in this disease suggested a synergistic effect between the two agents, with gemcitabine causing the persistence of treosulfaninduced DNA interstrand crosslinks (4).

During the period of this retrospective study, New Cross Hospital, Wolverhampton participated in the prospective randomised controlled trial of ATP-based tumour chemosensitivity assay (ATP-TCA)-directed chemotherapy vs. physician choice. This trial aimed to determine whether assay-directed chemotherapy can improve response rates and progressionfree survival in patients with recurrent platinum-resistant ovarian cancer who progressed within six months of the primary platinum-based therapy (5). One of the combinations explored was treosulfan and gemcitabine (TG). Within the assay-directed treatment group, partial/complete responses of $40.5 \%$ were observed, with a trend towards improved response rates and progression-free survival.

We undertook a retrospective case note review to examine response rates to TG, toxicity and the impact on survival in patients with platinum-resistant ovarian cancer. 


\section{Materials and methods}

Study design. A retrospective case-note review of patients with platinum-resistant ovarian cancer treated with TG was undertaken in a cancer centre between May 1st, 2000 and November 1st, 2005. Follow up occurred until February 28th, 2008.

Platinum resistance was defined as progression within six months of a platinum regimen.

Treatment regimen. Two TG regimes were administered during the study period. The 3 -weekly regime was treosulfan $5 \mathrm{~g} / \mathrm{m}^{2}$, administered intravenously (i.v.), over $30 \mathrm{~min}$ in $250 \mathrm{mls}$ normal saline and gemcitabine $1000 \mathrm{mg} / \mathrm{m}^{2}$ i.v. over $30 \mathrm{~min}$ in $250 \mathrm{mls}$ normal saline, both on day 1 . Gemcitabine was administered after the treosulfan. The 2-weekly regime was $5 \mathrm{~g} / \mathrm{m}^{2}$ oral treosulfan over 5 days with gemcitabine $1000 \mathrm{mg} / \mathrm{m}^{2}$ i.v. on day 1 .

The antiemetics used were i.v. 5HT3 antagonist and dexamethasone $8 \mathrm{mg}$ before chemotherapy, with an oral 5HT3 antagonist and dexamethasone $2 \mathrm{mg}$ orally 3 times/day for 3 days after chemotherapy. Oral domperidone 10-20 mg was also prescribed as required.

Patients were seen at 3-weekly intervals during chemotherapy and followed up 6- to 8-weekly after chemotherapy. They had a serum ca125 performed at each visit.

Outcomes. Complete response (CR) was defined as normal serum ca125 and normal computerised tomography (CT scan). Partial response (PR) was defined as a $>50 \%$ decrease in ca125 levels, with a second sample 28 days later, or a $50 \%$ decrease of measurable disease on the post-treatment CT scan, according to WHO criteria. Stable disease was defined as a $<50 \%$ decrease in ca125 levels or measurable disease on the CT scan, or no change in the levels and disease. Progression was the development of new lesions or an increase in ca125 or disease on the CT scan.

Overall survival time was measured from the date of diagnosis until the date patients succumbed to any cause, while relapse-free survival time was measured until the date of first relapse. Overall and relapse-free survival following the start of TG chemotherapy were measured from the date of commencement until the date patients succumbed to any cause and the date of first relapse, respectively. Survival times were censored at the end of the follow-up (February 28th, 2008).

Statistical methods. Estimates of cumulative survival were obtained using the Kaplan-Meier method and 95\% confidence intervals were constructed as appropriate.

\section{Results}

Forty-nine patients had TG chemotherapy for platinum resistant ovarian cancer at New Cross Hospital, Wolverhampton between May 1st, 2000 and November 1st, 2005. The median age at diagnosis was 55 years (range, 31-72), with the median age at the commencement of TG chemotherapy being 58 years (range, 34-79). Forty-six patients had epithelial ovarian cancer, 1 had a primary fallopian tube carcinoma and 2 had primary peritoneal cancer. The histological subtypes were as follows: 36 serous, 3 endometrioid, 1 clear cell, 6 mucinous
Table I. Stage distribution on diagnosis.

\begin{tabular}{lc}
\hline Stage & No. of patients \\
\hline $1 \mathrm{~A}$ & 1 \\
$1 \mathrm{C}$ & 6 \\
$2 \mathrm{~B}$ & 1 \\
$2 \mathrm{C}$ & 3 \\
$3 \mathrm{~A}$ & 3 \\
$3 \mathrm{C}$ & 30 \\
4 & 4 \\
Unknown & 1 \\
\hline
\end{tabular}

Table II. Pre-treatment of patients.

\begin{tabular}{cc}
\hline Prior platinum or paclitaxel regimens & No. of cases \\
1 & 17 \\
2 & 25 \\
3 & 6 \\
Prior non-platinum/paclitaxel regimens & No. of cases \\
1 & 11 \\
2 & 1 \\
\hline
\end{tabular}

${ }^{a}$ All were caelyx, except for two topotecan regimens.

Table III. Response rates stratified according to the line of treatment.

\begin{tabular}{lcrcc}
\hline Line of RX & CR & PR & SD & PD \\
\hline 2nd & 1 & 9 & 3 & 2 \\
3rd & 0 & 10 & 6 & 3 \\
4th & 0 & 6 & 5 & 2 \\
5th & 0 & 0 & 1 & 1 \\
\hline
\end{tabular}

$\mathrm{CR}$, complete response; $\mathrm{PR}$, partial response; $\mathrm{SD}$, stable disease; $\mathrm{PD}$, progressive disease.

and 3 unknown. The stage distribution on diagnosis is shown in Table I.

Fourteen patients $(28.6 \%)$ had bulky residual disease following surgery or biopsy, $19(38.8 \%)$ had minimal residual disease, $13(26.5 \%)$ appeared to have no residual disease and no information was available for 3 patients $(6.1 \%)$. Seven patients underwent interval debulking surgery during the study period.

Carboplatin single agent was used as first-line chemotherapy in $10(20.4 \%)$ patients and carboplatin and taxol in 39 patients (79.6\%). The time of response to first-line platinum-based chemotherapy was $<6$ months for $26(53 \%)$ patients, between 6 and 12 months for $10(20.4 \%)$ and $>12$ months for 13 patients (26.5\%). 


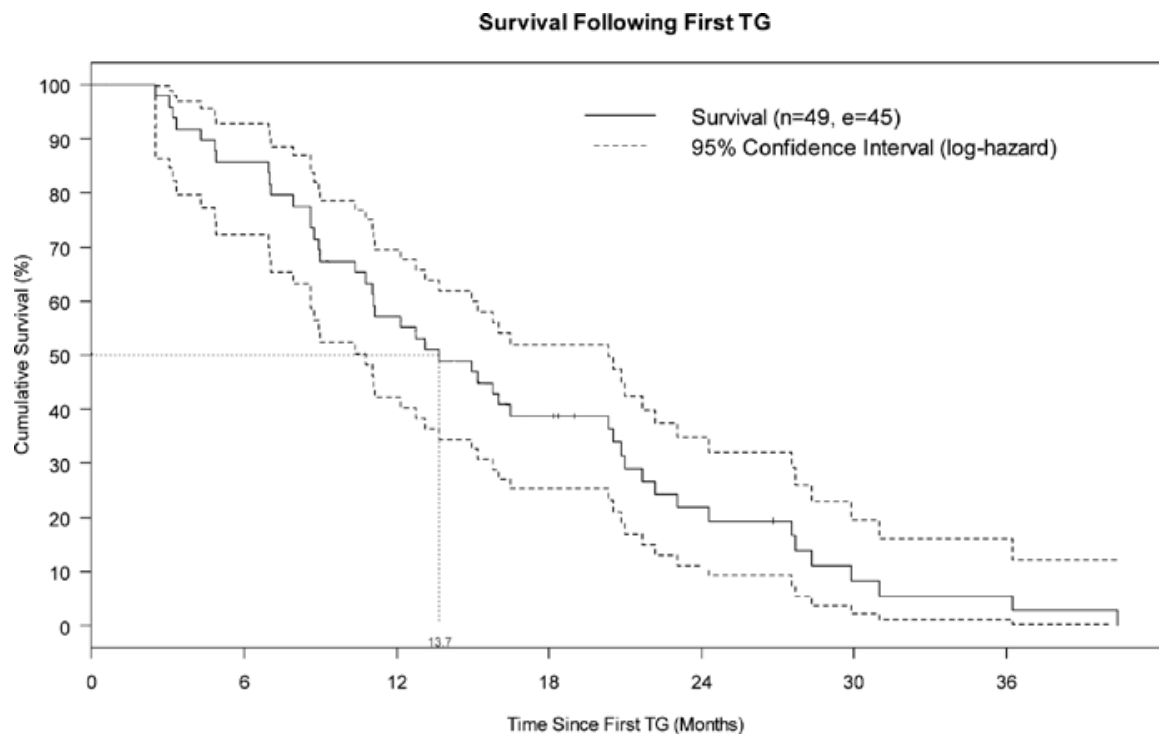

Figure 1. Survival following first TG. N, number of subjects; e, number of events.

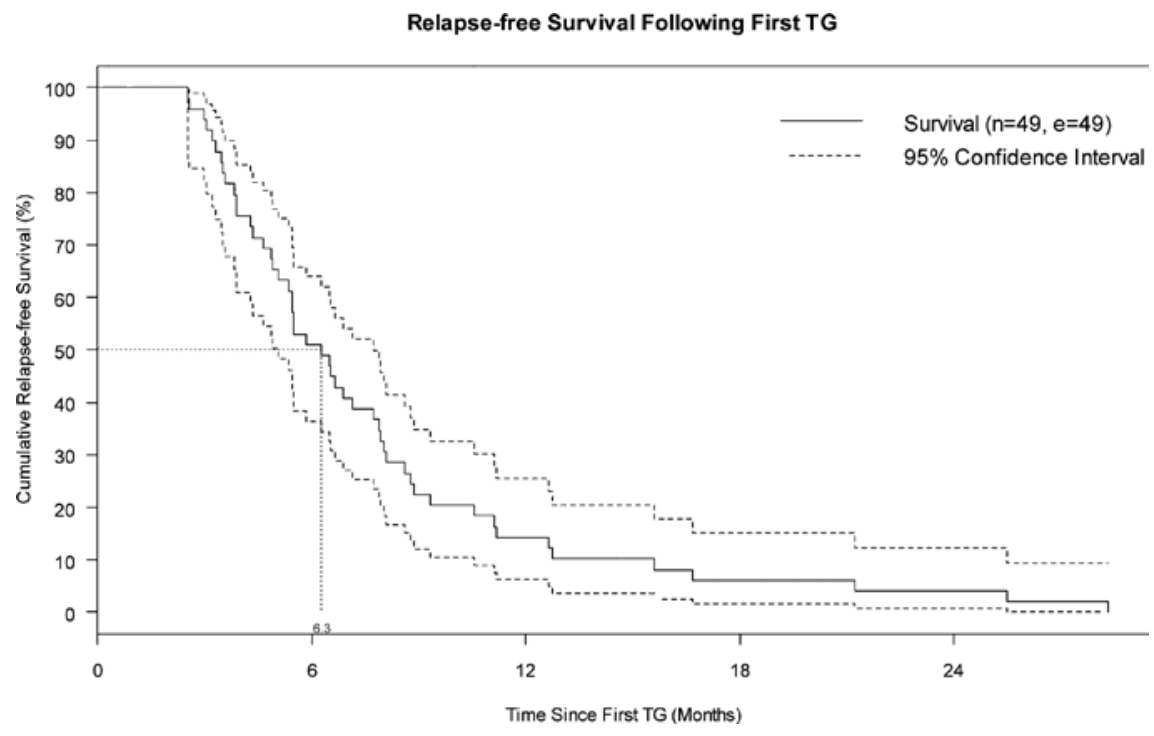

Figure 2. Relapse-free survival following first TG. N, number of subjects; e, number of events.

Sixteen patients took part in the ATP-TCA assay-directed vs. physician choice chemotherapy trial. Four patients were previously in the ICON 4 trial which investigated the use of second-line paclitaxel plus platinum chemotherapy vs. conventional platinum-only chemotherapy in patients with relapsed platinum-sensitive ovarian cancer.

Fourteen patients had the 2 -weekly TG regime, of whom 5 were switched to the 3 -weekly regime due to toxicity. In the latter part of the study the 3-weekly regime was used as it was better tolerated. The median number of cycles of TG given was 7 (range, 3-22 cycles).

Table II highlights the heavy pre-treatment of these patients with both platinum/paclitaxel-based chemotherapy and nonplatinum/taxane drugs.

On relapse, 15 patients received second-line TG chemotherapy with platinum-resistant disease, 19 third-line, 13 fourth-line and 2 patients received fifth-line treatment.
Fifteen $(30.6 \%)$ patients had stable disease after TG and $25(51 \%)$ had PR. One patient (2\%) had CR to TG when the second line was used. Eight patients (16.3\%) had progressive disease when the drugs were used in combination. Table III shows the response rates stratified according to the line of treatment.

Our study was retrospective and toxicity was poorly recorded. The median number of cycles given was 7 , implying this was a well-tolerated regime. Twenty-eight episodes of toxicity were recorded in 16 patients. Grade 4 neutropenia led to sepsis twice in one patient. The following episodes were recorded: myelosuppression (12), oedema (1), fatigue (9), myalgia (2), nausea (1), mouth ulcers (1), constipation (1) and diarrhoea (1). There were no treatment-related deaths.

Median follow-up was 45.1 months (range, 12.2-118.3). Forty-five of the 49 women succumbed to the disease before the end of the follow-up. The median survival was 45.1 months 
(95\% CI, 35.6-51.8). All 49 women relapsed at some point during the follow-up. The median relapse-free survival was 12 months (95\% CI, 10.7-6.4).

Median survival from the start of TG chemotherapy was 13.7 months (95\% CI, 10.8-20.3; Fig. 1). Relapse-free survival following the start of TG was 6.3 months (95\% CI 5.1-7.7; Fig. 2).

\section{Discussion}

The optimal therapy forpatients with relapsed platinum-resistant ovarian cancer is unknown. Strategies such as chemotherapy agents, chemotherapy with targeted biological agents and hormonal therapies can all be considered. Many factors come into play, such as patient wishes, previous chemotherapy exposure, patient performance status and comorbidities.

As a single agent, treosulfan has been shown to have limited activity in ovarian cancer. In a randomised controlled trial of first-line chemotherapy in patients considered unfit for cisplatin, single-agent treosulfan at a dose of $7 \mathrm{~g} / \mathrm{m}^{2}$ i.v. every four weeks was compared to single agent carboplatin (AUC 6) every four weeks (6). The response rate for treosulfan was only $29 \%$ compared to $49 \%$ for carboplatin $(95 \%$ CI, $5-35 ; \mathrm{p}=0.008)$, with a median time to progression of 10 months for carboplatin (95\% CI, 9-12) compared to 5 months for treosulfan (95\% CI, 4-6).

Gemcitabine has significant activity in ovarian cancer and has an acceptable toxicity profile. In phase I and II studies gemcitabine as a single agent had an overall response rate of $14-22 \%$, with a $30 \%$ rate of stable disease. The median duration of response ranged from 4 to 10.6 months in patients with heavily pre-treated and platinum-resistant ovarian cancer (7).

The combination of treosulfan and gemcitabine was first discovered to have pre-clinical and clinical activity in patients with platinum refractory recurrent ovarian cancer in studies using the ex vivo ATP tumour chemosensitivity assay, where a response rate of $48 \%$ was recorded (8).

Liposomal doxorubicin or single agent paclitaxel is the standard in the United Kingdom for patients who have progressed on platinum chemotherapy, following NICE guidance (9).

Pegylated liposomal doxorubicin has been compared, in a randomised controlled trial, to topotecan in the treatment of women with epithelial ovarian cancer recurrent or refractory to platinum-based chemotherapy. It was only in the platinumsensitive group that a significant survival benefit in favour of liposomal doxorubicin was noted (10). Hand-foot syndrome and stomatitis were experienced with liposomal doxorubicin, with $3.8 \%$ of patients having to discontinue the drug as a result of the cutaneous toxicity.

The use of topotecan in ovarian cancer has been subject to a systematic review (11). However, the studies were of poor methodological quality. Although effectiveness may be the same as paclitaxel or pegylated liposomal doxorubicin, topotecan has a significantly worse haematological side-effect profile.

Toxicity is a major consideration when patients are offered palliative chemotherapy for platinum-resistant ovarian cancer. Our study is retrospective and toxicity was poorly recorded. The median number of cycles given was 7, implying this is a well-tolerated regime. Possible side-effects from this regime include myelosuppression, nausea and vomiting, fatigue, skin rash, constipation, stomatitis, influenza-like symptoms, peripheral oedema and pulmonary toxicity. Grade 3-4 anaemia, leukopenia and thrombocytopenia were found in 5.1, 11.9 and $8.5 \%$ of patients, respectively, when treated with TG for recurrent ovarian cancer, albeit with gemcitabine administered on days 1 and 8 at a higher dose than in this series (12).

Acute myeloblastic leukaemia is a recognised secondary malignancy which can be induced by treosulfan use, with leukaemia developing 21-58 months (median 50) after treosulfan for the treatment of ovarian cancer (13). However, this malignancy type is unlikely to be a significant concern in platinum-resistant patients.

Trials investigating the combination of biological agents, such as bevacizumab and single-agent chemotherapy are showing promising results. This approach allows for the potential to reduce haematological toxicity in a heavily pretreated group of patients compared to chemotherapy doublets. However, other specific side-effects such as risk of bowel perforation will have to be considered $(14,15)$.

Our study included patients treated with TG when their disease progressed to platinum-resistant. This was a heavily pretreated group of patients, with both platinum/taxane-based agents, pegylated liposomal doxorubicin and topotecan. The response rate of $53 \%$, with a $30.6 \%$ rate of stable disease has never previously been reported in this group of patients. This response rate translated to a relapse-free survival of 6.3 months and a median survival time from the start of $\mathrm{TG}$ of 13.7 months.

In conclusion, given the activity of the treosulfan/ gemcitabine doublet in platinum-resistant ovarian cancer, a randomised controlled trial involving TG compared to pegylated liposomal doxorubicin should be conducted with the aim of investigating survival, toxicity and quality of life.

\section{References}

1. UK ovarian cancer statistics, 2008. Cancer Research UK website.

2. De Vita: Treosulfan and gemcitabine in heavily pretreated patients with breast and ovarian cancer: laboratory study and clinical pilot trial of the ISCO clinical study group. 7th edition, p1364, 2005.

3. Fennelly J: Treosulfan (Dihydroxybusulphan) in the management of ovarian carcinoma: Br J Obstet Gynecol 84: 300-303, 1977.

4. Corrie PG, Shaw J, Spanswick VJ, et al: Phase I trial combining gemcitabine and treosulfan in advanced cutaneous and uveal melanoma patients: Br J Cancer 92: 1997-2003, 2005.

5. Kurbacher CM, Grecu OM, Stier U, et al: ATP chemosensitivity testing in ovarian and breast cancer: early clinical trials: Recent Results Cancer Res 161: 221-230, 2003.

6. Reed NS, Poole CJ, Coleman R, et al: A randomised comparison of treosulfan and carboplatin in patients with ovarian cancer: a study by the Scottish Gynaecological Cancer Trials Group (SGCTG). Eur J Cancer 42: 179-185, 2006.

7. Lorusso D, di Stefano A, Fanfani F and Scambia G: Role of gemcitabine in ovarian cancer treatment: Ann Oncol 17: 188-194, 2006.

8. Grecu OM, Kurbacher CM, Mallman P, Bruckner HW and Cree IA Treosulfan and gemcitabine in heavily pre-treated patients with breast and ovarian cancer: Proc Am Soc Clin Oncol 20: abs. 2500, 2001.

9. Paclitaxel, pegylated liposomal doxorubicin and topotecan in the treatment of advanced ovarian cancer, relapsed disease. NICE guidance, 2005.

10. Gordon AN, Tonda M, Sun S and Rackoff W: Long-term survival advantage for women treated with pegylated liposomal doxorubicin compared with topotecan in a phase 3 randomized study of recurrent and refractory epithelial ovarian cancer: Gynecol Oncol 95: 1-8, 2004. 
11. Peng LH, Chen $X$ and Wu T: Topotecan for ovarian cancer. Cochrane Database Syst Rev: Apr 16, 2008. E-pub ahead of print.

12. Breidenbach M, Rein DT, Schondorf T, Schmidt T, Konig E, Valter M and Kurbacher CM: Hematological side-effect profiles of individualised chemotherapy regimen for recurrent ovarian cancer. Anticancer Drugs 14: 341-346, 2003.

13. Pederson-Bjergaard J, Nissen NI, Sorensen HM, et al: Acute nonlymphocytic leukaemia in patients with ovarian cancer following long-term treatment with treosulfan (dihydroxybusulfan). Cancer 45: 19-29, 1980.
14. Cannistra SA, Matulonis UA, Penson RT, et al: Phase II study of bevacizumab in patients with platinum-resistant ovarian cancer or peritoneal serous cancer. J Clin Oncol 25: 5180-5186, 2007.

15. Garcia AA, Hirte H, Fleming G, et al: Phase II clinical trial of bevacizumab and low-dose metronomic oral cyclophosphamide in recurrent ovarian cancer: a trial of the California, Chicago and Princess Margaret Hospital phase II consortia. J Clin Oncol 26: 76-82, 2008. 